\title{
De l'espace produit à l'espace vécu
}

"Nous avons quasiment perdu le pouvoir de rêver un monde où la parole soit prise et partagée, où personne ne puisse limiter la créativité d'autrui, où chacun puisse changer la vie.»

I. ILLICH

\section{Du productivisme à l'existentiel}

Cette remarque d'IVAN ILLICH est un constat d'échec qui situe fort bien la voie choisie par notre société technologique basée sur l'exploitation intensive des capacités humaines avec pour corollaire une société malade ayant perdu le goût de la liberté. Toute l'élaboration de notre cadre sociétal a reposé sur une finalité mensongère, à savoir que la recherche du profit maximum à travers une technologie hautement sophistiquée devenait le moyen indispensable à la recherche du «bien-être». Actuellement la révolte gronde contre les abus engendrés par une telle recherche. Les débuts sont certes timides, mais de plus en plus se généralise la prise de conscience de l'impact désastreux du système économique et politique que nous subissons car «l'homme avec la maîtrise de la nature, prend conscience de l'aliénation de son propre destin et de son travail $\left.(\ldots)^{11}\right)$. Dès les premières années, l'individu se trouve conditionné dans des structures qui lui sont étrangères et qui inhibent en lui tout désir/ pouvoir de création. Dans nos sociétés, la personnalité de l'individu s'estompe asservie qu'elle est aux banalités d'une quotidienneté tout entière tournée vers le profit et l'exploitation maximum de la personnalité humaine et qui, sans aller aussi loin que R. VACCA dans son ouvrage «Demain le Moyen Age»2), nous précipite irrémédiablement vers une période difficile, voire critique pour l'humanité.

C'est notamment à travers l'écologie que se reconnaissent et se regroupent les contestataires du système mis en cause. Le rejet d'une telle finalité productiviste fait problème car l'écologie devenant une mode, encore faut-il se méfier de «(...) la masse des prétendus "mouvements écologiques» dans les pays industrialisés qui insistent sur la morale personnelle du recyclage et de la non-consommation qui sont (...) des tactiques de diversion qui n'arrivent pas à mettre l'accent essentiellement sur la destruction du système de production axée sur le profit» 3 ). Cette remarque de J. GALTUNG est intéressante car elle explique comment la classe dominante peut, à travers ces «mouve- ments écologiques», canaliser le mécontentement qui se fait de plus en plus fort. Cependant la question essentielle est de savoir ce que recouvre le concept d'écologie et les modes d'action qu'il peut engendrer afin d'éviter de tomber dans les pièges tendus par l'idéologie dominante. C'est dans le cadre de l'écologie humaine, notre préoccupation essentielle, que nous désirons mettre en évidence les réflexions de CL. RAFFESTIN:

«L'écologie humaine propose un changement de projet social en apparaissant comme la critique d'un quotidien tout entier tourné vers l'appropriation et la production qui sont deux mouvements qui ne tiennent que très peu compte des conditions d'existence. A cet égard, le projet de l'écologie humaine est en opposition avec la rationalité technico-économique d'une part et la finalité productiviste d'autre part. Dans ces conditions, l'écologie humaine est davantage un projet scientifique qu'une science constituée»4).

A la suite de ces considérations et toujours selon le le même auteur, il convient de préciser que c'est l'action politique qui devient le moteur principal de ce projet scientifique. L'auteur utilise des concepts tels que créativité, liberté, autonomie qui deviendront les mots-clés de cette action politique qui devrait permettre de proposer un jour un modèle sociétal à l'intérieur duquel de tels concepts seront garantis par l'ensemble de la communauté. Il faut d'abord saisir que l'écologie ne peut être véritablement efficace comme moyen d'action que si elle est comprise d'abord comme une prise de conscience de chacun d'entre nous du système coercitif dans lequel nous vivons et ensuite comme un refus de ce système. La somme de ces comportements individuels qui vont à l'encontre de la rationalité technico-économique actuelle doit aboutir à une prise de conscience générale axée sur une nouvelle finalité, plus existentielle, qui rendrait caduque l'action des détenteurs du pouvoir actuel: «C'est en se libérant des servitudes du matérialisme, en stimulant la spontanéité, le paradoxe et l'intuition, moteurs de la création et en développant l'intellect, élément de base de la vie quotidienne que l'anti-mouton peut se développen ${ }^{5)}$. Voilà une nouvelle problématique qui dévoile de façon pertinente le fossé qui

Philippe Cordey, Département de géographie, rte des Acacias 18,1227 Genève-les-Acacias. 
existe entre les tenants de la conception actuelle bien généralisée d'une écologie mettant le poids sur la nonconsommation et celle que certains auteurs préconisent et qui relève encore de l'utopie. Rendre la réalité utopique, voilà déjà lancée une proposition de travail dont la première action devrait être de cerner les formes de notre appartenance et de notre conditionnement au système actuel, ceci afin de mieux pouvoir rompre ce lien, objet de dépersonnalisation, de soumission et d'aliénation.

La transcription spatiale de ce que nous venons d'écrire dans le cadre de l'écologie humaine peut se traduire par le rejet de participer à toute forme d'organisation supprimé relevant d'une finalité productiviste. De concept abstrait, l'espace est devenu par la praxis, l'action humaine, un élément important du mécanisme diabolique réglant les rapports entre les individus et basé sur la relation dominant/dominé à travers une contradiction fondamentale qui est l'essence même du capitalisme, à savoir la participation générale à un processus de production débouchant sur une appropriation privée. Sur le plan urbain, par exemple, l'utilisation actuelle de l'espace est l'obstacle majeur à la réalisation de l'homme dans la ville.

Dévoiler et dénoncer les arguments politiques et économiques qui sont à la base de toute urbanisation ou planification urbaine, telles doivent être, conformément à la définition de l'écologie donnée ci-dessus, les premiers actes de remise en cause du développement urbain axé toujours sur cette finalité du maximum de profit. Proposer un nouveau cadre de vie à l'échelle de l'individu et non plus de l'entreprise capitaliste, telle doit être la nouvelle formulation politique du contre-pouvoir urbain qui se dessine peut-être de nos jours. Pourquoi l'espace urbain revêt-il à nos yeux un intérêt tout particulier? H. LEFEBVRE6) explique justement qu'après la société industrielle et post-industrielle nous entrons dans l'ère de «l'urbain», de la «Révolution urbaine». En plus de cette affirmation essentielle, notre intérêt est lié au fait que la ville est le lieu de rassemblement des pouvoirs et qu'elle est, à une échelle réduite, la transcription spatiale et ponctuelle d'un ordre économique et politique contre lequel nous nous élevons. D'autre part, la ville devient le lieu d'action de luttes qui prennent de plus en plus d'ampleur. En effet, beaucoup de personnes commencent à percevoir le jeu dont elles sont les victimes. La spéculation, la démolition faisant place ensuite à la rénovation, la marginalisation de certains espaces, l'expropriation, le prix des loyers, les bruits, les nuisances de toutes sortes, la solitude des grands ensembles et les comportements agressifs qui s'en dégagent, tels sont brièvement esquissés les éléments moteurs d'une nausée générale qui peut, dans certains cas, prendre figure de lutte ouverte face à l'ordre établi. Cependant, l'efficacité de ces luttes urbaines ne sera véritable que lorsqu'elles mettront l'accent sur la proposition d'un nouveau cadre existentiel. Au-delà d'une pratique à court terme nécessaire et souvent efficace (grève des loyers, occupation d'appartements vides, information auprès de public, etc...), il convient d'aller à la recherche de cette utopie que nous énoncions auparavant à travers notamment la participation de l'individu à l'élaboration du paysage urbain dans lequel il vit.

\section{Du pouvoir dominant au contre-pouvoir urbain}

La finalité essentielle dans cette logique du discours écologique est, sur le plan urbain, de dénoncer les mécanismes d'appropriation et d'aménagement de l'espace qui sont à la base de son utilisation différentielle. Avant d'analyser la façon dont les groupes dominants agissent, il convient de les connaître. M. GUILLAUME définit ces groupes de la façon suivante: «(...) la classe dominante est la classe sociale qui s'assure la maîtrise des mécanismes ećonomiques et des mécanismes du pouvoin ${ }^{7)}$. Autant dire qu'il s'agit, en plus de l'Etat, des partis politiques et des syndicats, des entreprises capitalistes: banques, compagnies d'assurances et autres sociétés financières, commerciales et industrielles. C'est la collusion de ces deux types de pouvoirs (économique et politique) qui est à la base de l'appropriation et de l'aménagement urbain. En effet, la différenciation de l'utilisation de l'espace urbain ne peut être saisie que par rapport à la société qui utilise cet espace de manière différentielle suivant les époques. Cette société comporte en elle des inégalités socioéconomiques qui font que certains peuvent avoir une action sur l'espace et d'autres pas. Les premiers appartiennent aux groupes dominants responsables de l'espace aménagé alors que les autres appartiennent à la catégorie de ceux qui subissent l'espace, c'est-à-dire les groupes dominés participant au processus de production de l'espace, mais non à son appropriation. Qui sont les groupes dominants et quels sont leurs moyens d'action sur l'espace urbain? A ces deux questions, nous répondrons par un schéma, celui de la figure 1.

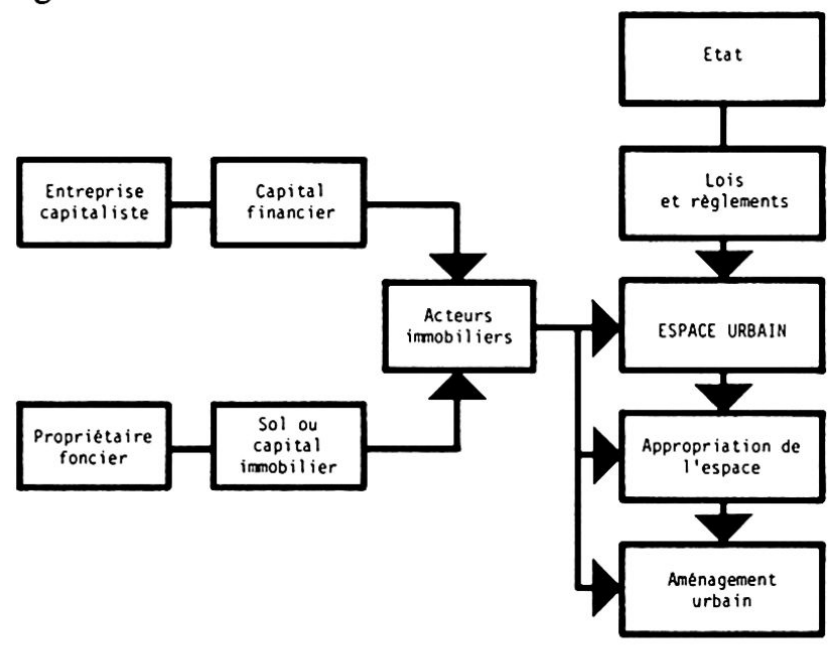

Ce schéma synthétise les "acteurs» en présence dans l'édification du paysage urbain. Les entreprises capi- 
talistes (sociétés financières, banques, assurances, etc....), si elles n'ont pas un rôle direct sur l'espace (rôle tenu uniquement par les acteurs immobiliers: promoteurs, constructeurs, architectes, etc....) n'en n'ont pas moins une importance considérable puisque ce sont elles qui vont avancer l'argent nécessaire aux acteurs immobiliers. Quant au propriétaire foncier, son rôle se réduit toujours à ce que MARX écrivait, à savoir un rôle passif lui permettant cependant d'accumuler le maximum de profit que son terrain peut lui procurer. Le rôle de l'Etat se divise en deux parties: il est d'abord acquéreur de terrains (donc propriétaire foncier) afin de les équiper pour les rendre constructibles. Il est ensuite régulateur de l'action des groupes dominants en offrant un "espace de règles» dans lequel se meuvent les acteurs immobiliers. Nous incluons donc l'Etat dans les groupes dominants car n'ayant pas les moyens financiers suffisants pour s'opposer à la politique de profit des groupes privés, il leur abandonne l'espace urbain et son aménagement.

La finalité productiviste de ces groupes s'exprime à travers les processus d'appropriation et d'aménagement de l'espace qui s'est matérialisé à partir de l'instant où il s'agissait de développer l'idée que la ville n'était que «l'image que la classe dominante prétend se donner d'elle-même et désire diffuser à l'extérieur ${ }^{8)}$. L'action de ces groupes dominants fut de tous temps régie par leur rationalité technico-économique qui, par la recherche du profit maximum permet d'atteindre l'objectif primordial du capitalisme: la croissance économique qui trouve sa finalité dans cette recherche du bien-être que nous énoncions plus haut. Comment se traduit sur l'espace urbain l'action de ces groupes? C'est à travers les mécanismes d'appropriation et d'aménagement urbains que nous tenterons de répondre à cette question.

\section{Appropriation de l'espace et rente foncière}

Les jeux d'aménagement de l'espace urbain sont liés à l'appropriation de l'espace qui se traduit par l'achat, la vente, la location de terrains et d'immeubles, en présence notamment de spéculateurs (intermédiaires) et de propriétaires fonciers qui essaient de tirer un maximum de profit des terrains qu'ils offrent aux promoteurs, architectes et autres constructeurs qui se chargeront de l'édification du paysage urbain. Cette appropriation de l'espace, qui doit ensuite déboucher sur son aménagement, est liée au problème de la rente foncière. Nous n'avons pas l'intention de refaire l'historique d'un tel concept en analysant les théories qu'il recouvre. Nous tenterons de schématiser cette notion que nous aborderons par la pensée marxiste tout en expliquant brièvement pourquoi nous rejetons en grande partie les théories "modernes» de l'organisation spatiale. Reprenons d'abord la théorie classique de la rente foncière agricole à partir des écrits de MARX et de RICARDo d'où nous tirons en grande partie le schéma de la figure 2.9)
RENTE FOHCIERE CHEZ MARX ET RICARDO

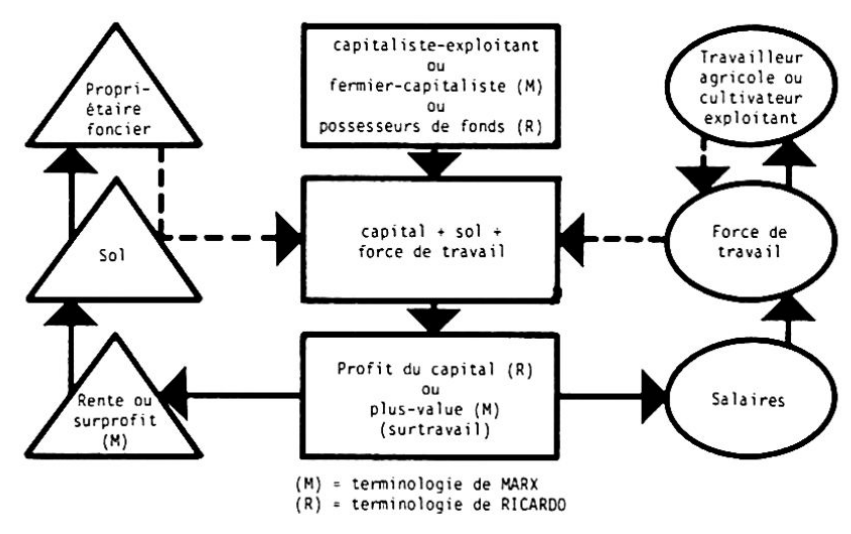

La rente agricole perçue par le propriétaire foncier est donc, chez MARX et RICARDo une part de la plusvalue perçue par le fermier-capitaliste, la plus-value se formant "quand l'ouvrier produit plus de valeur qu'il n'en coûte»10). Le montant de la rente est alors fixé par le «profit moyen que le capital rapporte dans les branches non agricoles (...)»11). Faute de place, nous n'aborderons pas les différentes rentes agricoles que MARX a mises en évidence. Nous analyserons donc immédiatement la rente foncière urbaine. K. MARX et D. RICARDO n'abordent pas ce problème de façon exhaustive. Le premier effleure le problème en reprenant l'idée d'A. SMITH selon laquelle la rente des terrains non agricoles est régie par la rente agricole. D. RICARDO, quant à lui, écrit simplement que les «loyers du sol sont en général le plus haut possible dans la capitale et surtout dans ces quartiers recherchés où il se trouve y avoir la plus grande demande de maisons, quelles que puissent être les causes de cette demande, soit raison de commerce et d'affaires, soit raison d'agrément et de société, ou simplement affaire de mode et de vanité» 12 ). La rente foncière en milieu urbain est fort complexe et de nombreux spécialistes abordent ce problème en prenant très souvent comme point de départ VON THUNEN qui a étudié les liens entre la ville et son umland. Avec des hypothèses de départ fort contestables et reprises par les théoriciens modernes de l'économie spatiale (plaine uniforme, égale densité, égale fertilité, présence d'un seul centre urbain), VON THUNEN montre comment les terrains proches de la ville deviennent objets de convoitise pour les agriculteurs.

La plus grande critique faite aux théories classiques relève du fait qu'elles n'abordent le problème de la rente foncière urbaine que sous l'angle de la production en négligeant complètement la demande de terrain sur le marché. Pour les économistes modernes, il faut considérer la rente comme le prix du sol qui se détermine à travers l'offre et la demande du sol. Cette critique, justifiée au premier abord, l'est moins si l'on considère les acteurs de la demande et de l'offre sur le marché foncier. Du côté de l'offre, les milieux immobiliers, promoteurs, constructeurs et autres spéculateurs mettant le terrain à la disposition des deman- 
deurs, à savoir les grandes firmes capitalistes d'une part, les consommateurs particuliers d'autre part. Le poids de la demande de ces derniers est absolument nul face à la puissance des groupes économiques. Il y a collusion entre les offreurs et une partie des demandeurs (entreprises) qui se fait au détriment des demandeurs particuliers (prix des loyers élevés, rejet dans des zones périphériques à cause de la tertiarisation du centre-ville pour des raisons de situation, de facilités d'accès, de prestige, etc....). Sans moyens financiers, sans moyens de pression, la classe dominée n'est pas apte à faire un choix ou à voir ses demandes prises en considération. D'autres critiques peuvent être faites aux théoriciens tels que W. ALONSO, L. WINGO, R. MAYER qui énoncent des hypothèses tout aussi insatisfaisantes ne tenant entre autres pas compte de l'attrait du site (A.-S. BAILLY) et du comportement des individus qui vont choisir tel ou tel quartier en fonction de critères totalement subjectifs qu'eux-mêmes ont de la peine à saisir et qui ne relèvent pas nécessairement d'une réflexion sur le prix des terrains, les facilités d'accès ou les coûts de transport. D'autre part, les hypothèses justifiant un espace continu et l'atomicité du marché (chez ALONSO par exemple) ainsi que celles énonçant que, puisque le prix du terrain va en augmentant de la périphérie au centre de la ville, les populations vont alors occuper dans les zones suburbaines des espaces plus grands à moindre coût, sont tout aussi aberrantes et nous nous réservons le droit de revenir une autre fois sur ce sujet qui demanderait un trop long développement. Nous aborderons en conclusion de cette partie l'analyse de la rente foncière urbaine à travers la pensée marxiste et selon trois postulats d'importance que l'analyse moderne tend à laisser de côté:

1. le poids énorme qu'exerce le facteur «situation» à l'intérieur d'un espace fortement urbanisé.

2. l'exploitation du progrès social de la part des groupes immobiliers spéculateurs, promoteurs, constructeurs et autres profiteurs d'un espace limité sur lequel une population augmente régulièrement.

3. la collusion entre le capital immobilier et le capital industriel, financier et commercial.

En résumé, et selon l'analyse marxiste, on peut écrire, à la suite de F. ALQUIER ${ }^{13)}$, que la rente urbaine provient de trois sources:

- des terrains qui permettent aux propriétaires fonciers d'empocher une rente grâce notamment au facteur situation.

- des acteurs immobiliers qui investissent sur les terrains en question pour augmenter leur profit.

- du propriétaire foncier qui, par son action, fait naître la rente absolue, c'est-à-dire qu'il peut retirer son terrain du marché de la production jusqu'à ce que les conditions économiques soient meilleures.

Toujours selon le même auteur, la rente foncière urbaine n'apparaît pas directement. Elle est inclue dans le prix du loyer qui, lui-même, se compose de l'amor- tissement de la construction et du taux d'intérêt du capital investi dans la construction et de la rente foncière.

En conclusion, et toujours en partie selon F. ALQUIER, la rente foncière urbaine dépend de cinq facteurs essentiels:

- la situation (accessibilité au centre par exemple).

- la "constructibilité» pour reprendre un terme de F. ALQUIER (ou coefficient d'occupation des sols).

- l'attitude du propriétaire foncier (notamment dans le cas de la rente absolue).

- l'attitude des constructeurs, promoteurs et entrepreneurs qui se font concurrence pour acquérir les terrains à bâtir.

- «l'environnement social qui est l'idée que se font les divers acheteurs de la nature socio-professionnelle des quartiers» ${ }^{14}$.

Ce dernier point est important car il peut influencer grandement les autres points cités. En effet, la rente d'un quartier proche du centre-ville peut demeurer faible si les habitants concernés sont d'un milieu ouvrier. Il suffit de démolir ce quartier pour revaloriser le sol sur lequel vont s'élever des immeubles neufs occupés par une population originaire d'une autre couche sociale, à revenus plus élevés. Le schéma, inspiré en partie de F. ALQUIER ${ }^{15)}$ devient tel qu'il est présenté à la fig. 3 .

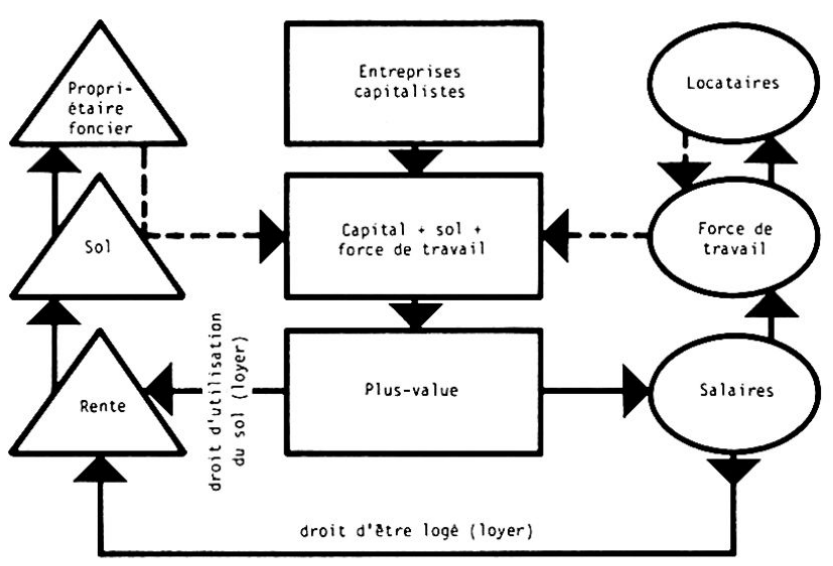

Il n'y a pas de contrat entre le propriétaire foncier et l'entrepreneur capitaliste (comme cela se passe dans la rente agricole). Le seul contrat qui puisse exister à un moment donné est un bail à la construction passé entre le propriétaire et le promoteur immobilier (droit d'utilisation du sol).

\section{Les coûts de l'aménagement urbain}

La transformation progressive de certains quartiers ou leur marginalisation par dégradation volontaire en vue d'une rénovation future sont deux des techniques utilisées par les acteurs immobiliers et l'Etat en vue de dégager de nouvelles sources de profit pour l'économie. 
Ces techniques, liées en cela à l'utilisation des effets d'une rationalité technico-économique antérieure s'expriment à travers les moyens suivants:

\section{- sur le plan morphologique}

dégradation progressive des immeubles (absence volontaire d'entretien ou de rénovation)

démolition des immeubles devenus inhabitables

création de parkings sur les espaces ainsi libérés, ce qui permet une nouvelle source de profit dans l'attente de la reconstruction du quartier ou d'une partie de celui-ci, voire d'un ilôt.

- sur le plan de la population

expulsion des habitants

remplacement de la population expulsée par des personnes qui acceptent de vivre dans des conditions parfois déplorables et qui seront facilement délogeables le moment venu (étrangers, jeunes, vieillards).

- sur le plan des activités

disparition progressive de l'accès aux commerces locaux qui ne sont pas remplacés

déplacement des artisans ou des industries plus importantes dans des zones industrielles situées à la périphérie de la ville.

L'aménagement du sol urbain d'après les acteurs immobiliers est lié fortement à la formation du prix du terrain qui est fonction à la fois des facteurs de situation, d'accessibilité au centre, de "constructibilité», de qualité des sols, mais aussi de la croissance économique de la ville en question. Si le sol en question est destiné à être occupé par des entreprises tertiaires, le prix sera élevé parce que ces firmes sont seules capables de payer un montant élevé pour jouir de certains privilèges. Si nous prenons le cas d'une classe sociale aisée, celle-ci acceptera également de payer un loyer élevé pour son logement si cela lui permet «de ne pas cohabiter avec n'importe qui». On en arrive ainsi à une «spécialisation» de l'espace urbain dont le prix n'est que le reflet d'un type d'activité ou de résidence bien précis. La ville devient alors un agrégat de "ghettos»: ghettos industriels et commerciaux (zone industrielle, centre-ville) et ghettos résidentiels (zones de villas, grands ensembles, quartiers dégradés, etc. ...): «Les gens de haut standing par les revenus ou le pouvoir en viennent à s'isoler eux-mêmes dans des ghettos de la richesse»16). Cette transformation progressive tend à fabriquer des espaces standardisés aussi bien au niveau des formes que des techniques utilisées, images des nouvelles perspectives morphologiques et économiques de notre temps. Cette transformation se fait au profit d'une tertiarisation toujours plus grande avec pour corollaire le rejet de la population dans des zones périphériques.

En ce qui concerne la seconde action (marginalisation) nous pouvons écrire que dans une ville, il est rare de ne pas trouver des zones dégradées, vétustes, des quartiers populaires dont le délabrement des habitations s'aggrave régulièrement sans que les pouvoirs publics n'interviennent pour exiger la rénovation ou l'entretien des immeubles. Etant eux-mêmes propriétaires d'un certain nombre d'habitations, ils sont ainsi également directement responsables de l'état de ces quartiers qui feront en réalité l'objet d'une rénovation future qui suppose d'abord la démolition complète de toutes les habitations. Le rôle futur de ces espaces est de donner une nouvelle aire d'extension aux activités tertiaires qui ne trouvent plus de place dans le centreville. Il s'agit alors d'élargir ce dernier en absorbant des quartiers qui ne répondent pas aux normes actuelles du développement économique. En conséquence, ces anciens quartiers doivent disparaître et afin d'éviter des réactions populaires et des frictions politiques, "on mise sur la progressive inadaptation du quartier, son asphyxie lente, puisqu'il ne répond plus aux exigences de la plus-value et du capital (...), bloquée par sa situation de non rentabilité»17).

L'expulsion des anciens habitants dans des zones périphériques où les coûts sociaux et économiques sont élevés (loyers plus élevés, temps de déplacements plus grands, fatigue, solitude, destruction du milieu familial, etc....), le relogement du quartier par des étrangers que l'on pourra facilement expulser le moment venu mais qui acceptent de vivre dans des conditions déplorables (manque d'hygiène, par exemple) à cause du bas niveau des loyers, le déplacement des petits artisans dans des zones industrielles où ils perdent leur situation de centralité tout en payant des loyers plus élevés, la disparition progressive des commerçants qui ne sont plus remplacés, la politique de rachat des terrains par l'Etat qui les mettra plus tard à la disposition de l'économie, la progressive dégradation des immeubles déjà citée, telles sont brièvement esquissées les techniques des pouvoirs publics et économiques. Les conséquences d'une telle politique sont nombreuses et néfastes pour une grande partie des gens concernés:

- déplacement dans des grands ensembles où l'anonymat est de rigueur

- temps de déplacements domicile-travail plus élevé

- fatigue plus grande

- coûts plus élevés (moyens de transports, loyers, etc....)

- difficulté d'adaptation avec les conséquences physiques et psychiques que cela suppose

- perte de l'«adhérence» à l'espace urbain, à la ville elle-même

- rejet des artisans dans des zones industrielles ayant pour conséquence la perte de la situation centrale antérieure, donc une perte de clientèle, des coûts supérieurs (transports plus longs, loyers plus élevés, etc....)

- apparition en ville d'un nouvel espace correspondant à la rationalité des groupes dominants: loyers élevés, activités tertiaires, boutiques de luxe, banques, etc.... 
Cette rénovation future peut dans certains cas provoquer l'opposition d'une grande partie de la population des quartiers en question, voire de la ville toute entière. Cette opposition est justifiée par le désir de conserver des espaces à l'intérieur desquels s'est développé un certain cadre de relations qui contraste fortement avec la monotonie des formes des autres quartiers ou des grands ensembles et la solitude humaine ainsi que l'anonymat qui s'en dégagent. L'étude de ces oppositions qui se font de jour en jour plus violentes est justifiée également par le souci de vivre dans un cadre urbain plus humanisé, ce qui pose le problème de la participation de l'individu au modelé de l'espace urbain dans lequel il vit. A cette occasion, citons S. RIMBERT qui écrit à ce sujet: «(...) il est commode au technocrate d'arguer de l'ignorance de l'homme de la rue pour lui imposer des formes standardisées. Cette attitude autoritaire et parfois arbitraire n'est qu'une solution de facilité dont le principe est déplorable, (...). Mettre l'urbanisme à la portée du peuple est une tâche civique et éducative (...). Loin de perdre leur temps, les architectes trouveraient dans les contacts avec les habitants et les usagers de quoi innover tout en s'appuyant sur la personnalité de la région: ils réussiraient peut-être à échapper aux canons stérilisants de la construction passe-partout dite internationale. Mais l'enseignement rapporte beaucoup moins qu'un contrat»18).

Ces modalités d'appropriation et d'aménagement de l'espace urbain provoquent l'apparition de coûts importants qui entravent aussi bien le genre de vie que la santé physique et psychique des individus concernés. En définitive, la finalité productiviste se traduit sur l'espace urbain par une appropriation et un aménagement qui tendent à faire des centres urbains des immenses concentrations tertiaires qui recherchent continuellement de nouveaux terrains d'expansion en rejetant la population dans des quartiers périphériques. Cette vérité connue de tous doit une fois pour toute être combattue en proposant une nouvelle problématique de l'espace urbain basée sur une finalité existentielle où l'être humain serait au centre de l'édification du paysage urbain hors des schémas traditionnels chers aux capitalistes. Le rôle des luttes urbaines commencées doit tendre vers le rejet de cette finalité matérialiste en défendant les intérêts des citadins opprimés et en éliminant ce sentiment d'impuissance (absence de pouvoir) face aux puissances économiques et étatiques. Cette première étape doit se prolonger dans l'élaboration d'un projet existentiel dans lequel apparaîtrait les nouvelles formes de relations que l'homme pourrait entretenir avec l'espace, banissant l'aliénation et la dépersonnalisation au profit de nouveaux concepts basés sur le respect des libertés humaines.

\section{Références}

1) J. ATTALI, «La parole et l'outil», PUF, Coll. «Economie en liberté», Paris, 1975, p. 16.

2) R. VACCA, «Demain le Moyen Age», A. Michel, Paris, 1973.

3) J. GALTUNG, «Les limites de la croissance et la politique des classes», in «Espaces et Sociétés», No 10 et 11, oct. 1973-fév. 1974, p. 162.

4) CL. RAFFESTIN, «Remarques sur le concept d'Ecologie Humaine», in International meeting on human ecology, Vienna, 1975, Georgi Publishing Company, p. 406.

6) R. ABREZOL, «L'anti-mouton humain», Ed. l'Age d'Homme, Lausanne, 1976, p. 66.

6) H. LEFEBVRE, «La Révolution urbaine», Gallimard, Coll. Idées, St-Amand, 1970, p. 13.

7) M. GUILlaume, «Le capital et son double», PUF, Coll. «Economie en liberté», Vendôme, 1975, p. 123.

8) H. CAPEL, «L'image de la ville et le comportement spatial des citadins», in «L'Espace Géographique», No 1, 1975, p. 79.

9) K. MARX, «Le Capital», Ed. populaire par J. BORCHARDT, PUF, Paris, 1965.

D. RICARDo, «Des principes de l'économie politique et de l'impôt», Flammarion, Paris, 1971.

10) M. DE MEURON, D. NOEL, W. SPIRIG, «Groupe rente foncière 74», EAUG, Genève, 1974, p. 6.

11) K. MARX, op. cit., p. 437.

12) D. RICARDO, op. cit., p. 178.

13) F. ALQUIER, «Contribution à l'étude de la rente foncière sur les terrains urbains", in "Espaces et Sociétés», No 2, mars 1971, pp. 83 à 85.

14) J.J. GRANELLE, "Espace urbain et prix du sol», Sirey, Paris, 1970, pp. 238-239.

15) F. ALQUIER, op. cit.

16) H. LEFEBVRE, «Le droit à la ville», Ed. Anthropos, Paris, 1968, p. 99.

17) M. MAIA, F. MEYER, M. VINCENT, «Le logement et la plus-value», EAUG, Genève, 1974, p. 21.

18) S. RIMBERT, «Les paysages urbains», A. Colin, Paris, 1973, pp. 235-236. 